\title{
Strain diversity in neurodegenerative disease: an argument for a personalized medicine approach to diagnosis and treatment
}

\author{
Amanda L. Woerman ${ }^{1}$ (D) \\ Received: 13 April 2021 / Revised: 13 April 2021 / Accepted: 14 April 2021 / Published online: 23 April 2021 \\ (c) The Author(s), under exclusive licence to Springer-Verlag GmbH Germany, part of Springer Nature 2021
}

The protein misfolding mechanism of disease was first described almost 40 years ago in a group of neurodegenerative disorders caused by the prion protein $(\operatorname{PrP})$ [1]. In these diseases, misfolding of cellular $\operatorname{PrP}\left(\operatorname{PrP}^{\mathrm{C}}\right)$ into a $\beta$-sheet-rich conformation $\left(\mathrm{PrP}^{\mathrm{Sc}}\right)$ results in $\mathrm{PrP}^{\mathrm{Sc}}$ serving as a template for additional $\operatorname{PrP}^{\mathrm{C}}$ misfolding. Recent advances in cryoelectron microscopy (cryo-EM) have led to high-resolution structures for several misfolded protein aggregates isolated from brain tissue, including the $\operatorname{PrP}^{\mathrm{Sc}}$ isolate 263K [2]. This model shows that the $\mathrm{PrP}^{\mathrm{Sc}}$ proteins are stacked in register, with the $\mathrm{R}$ groups from each amino acid in the plane of the $\mathrm{C}_{\mathrm{a}}$ backbone. The amides of the backbone are perpendicular to the plane, facilitating hydrogen bonds between protein layers to stabilize the flat, misfolded structure. In this conformation, $\mathrm{PrP}^{\mathrm{Sc}}$ becomes a template for progressive misfolding of $\operatorname{PrP}^{\mathrm{C}}$, with each replication event creating a new layer of intermolecular hydrogen bonds. Presumably, this mechanism leads to fibril formation and progressive neurodegeneration in a variety of distinct disorders, including Creutzfeldt-Jakob disease (CJD), fatal familial insomnia, and Gerstmann-Straussler-Scheinker disease.

The prion hypothesis was initially viewed as incompatible with the observation that the disease agent (at the time thought to be a slow virus) was capable of inducing multiple disorders in the absence of a nucleic acid (discussed in [3]). This seeming conflict was resolved with the introduction of the strain hypothesis, which proposes that the disease a patient develops is determined by the distinct conformation $\mathrm{PrP}^{\mathrm{Sc}}$ misfolds into, rather than mutations in a viral genome [4-6]. In this review cluster, the article by Dr. Jason Bartz discusses our current understanding of $\operatorname{PrP}^{\mathrm{Sc}}$ strain biology in both human disease and other mammalian species, as well

Amanda L. Woerman

awoerman@umass.edu

1 Institute for Applied Life Sciences and Department of Biology, University of Massachusetts Amherst, Amherst, MA, USA as the host and non-host factors that impact strain evolution in an individual [7].

Over the last 2 decades, research on other neurodegenerative diseases, including Alzheimer's disease (AD) and multiple system atrophy (MSA), has increasingly shown that the protein misfolding mechanism is not exclusive to the conversion of $\operatorname{PrP}^{\mathrm{C}}$ into $\operatorname{PrP}^{\mathrm{Sc}}[8,9]$. Consistent with the growing experimental observations that proteins like $\beta$-amyloid $(\mathrm{A} \beta)$, tau, and $\alpha$-synuclein behave like prions, cryo-EM structures of recombinant fibrils or patient-derived protein aggregates containing these proteins all show in register templates, implying that the same mechanism that supports $\mathrm{PrP}^{\mathrm{Sc}}$ propagation also enables self-templating of pathogenic $\mathrm{A} \beta$, tau, and $\alpha$-synuclein (several of these structures are discussed in [10-12]). Along with the growing need to expand the use of the term 'prion' to apply to a larger group of proteins is the need to define and investigate the disease-causing strains that result from their misfolding. Four reviews in this cluster focus on the phenotypic heterogeneity that manifests as a result of variability across non- $\mathrm{PrP}^{\mathrm{Sc}}$ prion strains.

Lau et al. discuss the structural and biological data supporting the hypothesis that distinct $\mathrm{A} \beta$ strains give rise to specific spatiotemporal patterns and types of pathology in AD patients, which likely impacts the clinical presentation of disease [10]. A similar diversity among amyotrophic lateral sclerosis (ALS) patients is now thought to arise from strain differences across proteins associated with the disorder. Ayers and Borchelt review the literature supporting the role of distinct SOD1, TDP-43, FUS, and C9orf72 strains in sub-types of familial ALS [13]. Tauopathies are a large and diverse group of disorders, each associated with a particular type and distribution of tau inclusions in the brain. Like $\operatorname{PrP}^{\mathrm{Sc}}$, where each prion disease is caused by its own unique strain, structural studies report conserved tau fibril conformations across patients with particular disorders, as discussed by Vaquer-Alicea et al. [11]. For example, the same conformation of misfolded tau has been resolved from both familial and sporadic AD patients [14], but this 
differs substantially from fibrils isolated from Pick's disease patients [15]. Notable similarities are seen with the $\alpha$-synuclein prion strains responsible for synucleinopathies, including MSA. As we discuss in the review by Holec and Woerman, mounting structural, genetic, and biological data support the hypothesis that MSA is caused by $\alpha$-synuclein prions that are distinct from those found in patients with Lewy body diseases, including Parkinson's disease [12].

A consistent theme emerges from each of these reviews, which is that the role of strain biology in disease heterogeneity has profound impacts on diagnosing and treating neurodegenerative disease patients. To date, the field suffers from the lack of definitive diagnostic tests for living patients. As noted in two of the reviews, there are significant shortcomings with the positron emission tomography (PET) ligands currently available or in development for diagnosing disease [10, 11]. Known PET ligands are typically only able to detect a subset of disease-causing strains, but it is not always clear which subset they are even capable of recognizing. In addition, off-target binding, particularly for the first-generation tau PET ligands, adds to the complexity of differentiating between tauopathies by affected brain regions. The lack of strain specificity also has ramifications for treating neurodegenerative disease patients. For example, small molecules or immunotherapies that interact with the misfolded conformation are likely to be strain-dependent if the structure or availability of the binding site is altered across diseases.

The traditional prion literature is full of lessons about the need for strain-specific tools to support therapeutic development. Despite decades of effort, human CJD prions have never been successfully propagated in cell culture models. Instead, high-throughput screening is typically done using mouse-adapted prion strains that can propagate in cells, including RML, which was developed at Rocky Mountain Laboratories by passaging sheep scrapie in mice. Screening against RML led to the identification of a group of promising 2-aminothiazoles (2-AMTs) [16], including the compound IND24, which was ultimately shown to double the lifespan of mice inoculated with RML prions [17]. However, IND24 and the other 2-AMTs showed no efficacy in mice inoculated with human CJD prions [18], underscoring the need to use disease-relevant strains in drug discovery programs to identify compounds capable of treating human diseases. Cryo-EM studies increasingly reveal that the conformations of misfolded tau and $\alpha$-synuclein isolated from the human brain differ significantly from the conformations found in common research tools. The continued use of model systems that fail to reproduce the disease-causing strains in human patients may be hampering the success of drug discovery efforts for neurodegenerative diseases.

The 2-AMT program led to a second lesson about the need to understand the composition and evolution of prion strains. Rather than a prion strain being defined as a single conformation, the cloud or quasispecies hypothesis proposes that a strain exists as a group of substrains [19, 20]. Typically, dominant substrains outcompete the minor ones for access to $\mathrm{PrP}^{\mathrm{C}}$ as substrate. However, when propagation of the dominant substrain(s) is repressed, the minor ones can emerge to cause disease (discussed in more detail in [7]). This phenomenon resulted in the emergence of a drug-resistant strain of RML in mice treated with the compound IND24 [17]. It is currently unknown if non- $\mathrm{PrP}^{\mathrm{Sc}}$ prion strains also exist as clouds of conformations or quasispecies, but the possibility of drug resistance is one the field should consider in the event that this phenomenon is discovered for other misfolded protein strains.

The growing recognition that strains have a profound impact across neurodegenerative diseases suggests the field needs to move toward a personalized medicine approach for diagnosing and treating patients. One cannot help but wonder what impact our current inability to definitively diagnose disease in living patients has had on the outcome of clinical trials. In a world where we can identify the exact strain or strains spreading in a patient's brain and match them with an appropriate therapeutic, would we finally see successful clinical outcomes? Personalized medicine has revolutionized the way we diagnose and treat cancer patients, and its application in neurodegenerative disease could offer a similar path forward for patients suffering from diseases such as $\mathrm{AD}$ and MSA. For this to become a reality, we must invest in the development of strain-specific cell and animal models of disease, which will enable successful clinical translation of diagnostics and therapeutics.

The traditional prion literature offers many valuable lessons that are becoming increasingly important as we learn more about the contribution of strains to protein misfolding diseases. Our hope with this cluster is that we can stimulate new ideas about the biology underlying each disease and inspire innovative approaches that lead to the development of personalized medicine for neurodegenerative disease patients.

Acknowledgements ALW is supported by the CurePSP Foundation and the University of Massachusetts Amherst.

\section{References}

1. Prusiner SB (1982) Novel proteinaceous infectious particles cause scrapie. Science 216:136-144

2. Kraus A et al (2021) Structure of an infectious mammalian prion. biorXiv 12:130-165 
3. Ghaemmaghami S (2017) Biology and genetics of PrP prion strains. In: Prusiner SB (ed) Prion Diseases. Cold Spring Harbor Laboratory Press, pp 45-56

4. Bessen RA et al (1995) Non-genetic propagation of strain-specific properties of scrapie prion protein. Nature 375:698-700

5. Telling GC et al (1996) Evidence for the conformation of the pathologic isoform of the prion protein enciphering and propagating prion diversity. Science 274:2079-2082

6. Tanaka $\mathrm{M}$ et al (2004) Conformational variations in an infectious protein determine prion strain differences. Nature 428:323-328

7. Bartz JC (2021) Environmental and host factors that contribute to prion strain evolution. Acta Neuropathol. https://doi.org/10.1007/ s00401-021-02310-6

8. Goedert M (2015) Alzheimer's and Parkinson's diseases: the prion concept in relation to assembled $\mathrm{A} \beta$, tau, and $\alpha$-synuclein. Science 349(6248): 1255555

9. Prusiner SB (2017) An introduction to prion diseases. In: Prusiner SB (ed) Prion diseases. Cold Spring Harbor Laboratory Press, pp $1-29$

10. Lau HHC, Ingelsson M, Watts JC (2020) The existence of Abeta strains and their potential for driving phenotypic heterogeneity in Alzheimer's disease. Acta Neuropathol 18:239-342

11. Vaquer-Alicea J, Diamond MI, Joachimiak LA (2021) Tau strains shape disease. Acta Neuropathol 10:47-87

12. Holec SAM, Woerman AL (2020) Evidence of distinct $\alpha$-synuclein strains underlying disease heterogeneity. Acta Neuropathol 12:89-234
13. Ayers JI, Borchelt DR (2020) Phenotypic diversity in ALS and the role of poly-conformational protein misfolding. Acta Neuropathol 2:20-45

14. Fitzpatrick AWP et al (2017) Cryo-EM structures of tau filaments from Alzheimer's disease. Nature 547:185-190

15. Falcon B et al (2018) Structures of filaments from Pick's disease reveal a novel tau protein fold. Nature 561:137-140

16. Ghaemmaghami S et al (2010) Discovery of 2-aminothiazoles as potent antiprion compounds. J Virol 84:3408-3412

17. Berry DB et al (2013) Drug resistance confounding prion therapeutics. Proc Natl Acad Sci USA 110(44):E4160-E4169

18. Giles K, Olson SH, Prusiner SB (2017) Developing therapeutics for PrP prion diseases. In: Prusiner SB (ed) Prion diseases. Cold Spring Harbor Laboratory Press, pp 579-597

19. Collinge J, Clarke AR (2007) A general model of prion strains and their pathogenicity. Science 318:930-936

20. Weissmann $\mathrm{C}$ et al (2011) Prions on the move. EMBO Rep 12:1109-1117

Publisher's Note Springer Nature remains neutral with regard to jurisdictional claims in published maps and institutional affiliations. 